\title{
BEM simulation model for coupled heat, moisture and air transport through a multilayered porous wall
}

\author{
L. Škerget ${ }^{1}$, A. Tadeu ${ }^{2} \&$ J. Ravnik ${ }^{1}$ \\ ${ }^{1}$ Faculty of Mechanical Engineering, University of Maribor, Slovenia \\ ${ }^{2}$ Department of Civil Engineering, University of Coimbra, Portugal
}

\begin{abstract}
Unsteady coupled air, moisture and heat energy flow through a porous solid is simulated numerically using a boundary element model (BEM). The governing transport equations are written and solved for the continuous driving potentials, i.e. relative humidity, temperature and air pressure. The moisture redistribution inside a wall with capillary active interior insulation is analysed to illustrate the applicability and accuracy of the proposed numerical model.

Keywords: boundary element method, heat and moisture transport, multilayered porous solid.
\end{abstract}

\section{Introduction}

Exterior building elements are exposed simultaneously to differences in temperature, moisture and air pressure, which have a major impact on the sustainability of the building. In this paper, the three-coupled transient HAM transport equations are solved using a boundary element numerical model (BEM) [9]. The singular boundary-domain integral representations of the basic conservation differential laws are based on the use of an appropriate fundamental solution that incorporates some of the physics of the transport phenomenon, such as accumulation and diffusion of the field function. However, the BEM leads to a fully populated system of equations. This can be efficiently overcome by the subdomain or macro element approach, which yields a sparse system similar to the domain type numerical models [7], while maintaining the accuracy and stability of the numerical algorithm. The discretization used in this paper leads to an overdetermined system of equations $[12,13]$. 
Next, the problem is briefly described. The relevant governing differential equations for energy, moisture and air transport are considered and formulated for the continuous driving potentials, i.e. relative humidity, temperature and air pressure. The corresponding singular integral representations are then developed, describing nonlinear heat, moisture and air transport in an integral form. One test benchmark example involving the moisture redistribution inside a multilayered porous wall with capillary active interior insulation, is given to illustrate the efficiency and accuracy of the proposed solution strategy [2, 3].

\section{Governing equations for a two-phase system}

Consider a two-component, two-phase thermodynamic system in a solution domain $\Omega$ bounded by a control surface $\Gamma$, where the indices $w, a$ and $m$ represent the water, air and dry porous material, and the indices $l$ and $v$ represent the two water phases. That is, $l$ refers to the liquid water and $v$ to the vapour water in a liquid/vapour moisture system.

\subsection{Moisture transport equation}

After some matematical manipulations the following moisture transport equation $[1,2]$ can be formulated

$$
\theta \frac{\partial \varphi}{\partial t}=\vec{\nabla} \cdot\left(D_{\varphi} \vec{\nabla} \varphi+D_{T} \vec{\nabla} T-\frac{1}{R_{w}} \frac{p_{s}}{T} \vec{v} \varphi-D_{l} \rho_{l} \vec{g}\right),
$$

where $\theta=d W / d \varphi$ is the slope of the sorption isotherm $W=W(\varphi)$. The primitive variable in eq. (1) is the relative humidity field function $\varphi\left(r_{j}, t\right)$, while the second, third and fourth terms on the right side of equation act as nonhomogeneous nonlinear source terms due to temperature gradient, vapour convection and gravity force. Note that, due to the second and third terms, eq. (1) is explicitly coupled to the heat energy transport equation and pressure equation. The transport coefficients $D_{\varphi}$ and $D_{T}$ are given as:

$$
D_{\varphi}=\delta_{p} p_{s}+D_{l} R_{w} \rho_{l} \frac{T}{\varphi} \quad \text { and } \quad D_{T}=\delta_{p} \frac{d p_{s}}{d T} \varphi+D_{l} R_{w} \rho_{l} \ln (\varphi)
$$

where the transport properties $\delta_{p}$ and $D_{l}$ stand for the vapour and liquid permeability of a solid material [14].

\subsection{Heat energy transport equation}

The heat energy balance equation considers accumulation within the control volume, energy flux (sensitive, latent and convective), in and out of the control volume and heat source/sink term [1-3], which leads to

$$
c_{e f f} \frac{\partial T}{\partial t}=\vec{\nabla} \cdot\left[\lambda_{e f f} \vec{\nabla} T-h_{l a t} \vec{n}_{v}-\rho_{a}\left(c_{p a}+\omega c_{p v}\right) \vec{v} T\right]+I,
$$


where the primitive variable in eq. (3) is the temperature field $T\left(r_{j}, t\right)$, whilst the coefficient $c_{e f f}=\rho_{m} c_{p, \text { eff }}$ is the effective specific heat per unit volume.

\subsection{Airflow dynamic equations}

The elliptic Poisson pressure equation governs the airflow through a porous solid is given by

$$
\frac{\partial}{\partial x_{j}}\left(\delta_{a} \frac{\partial p}{\partial x_{j}}\right)=0 \quad \text { and } \quad \rho_{a} v_{j}=-\delta_{a} \frac{\partial p}{\partial x_{j}},
$$

with $\delta_{a}$ is the air permeability of a solid matrix, where the time dependence of the two field functions, velocity and pressure, account for the effect of time dependent pressure boundary conditions.

\subsection{Initial and boundary conditions}

The initial conditions in general represent the distribution of field functions in the solution domain and their normal derivatives on the boundary and are given by the relations

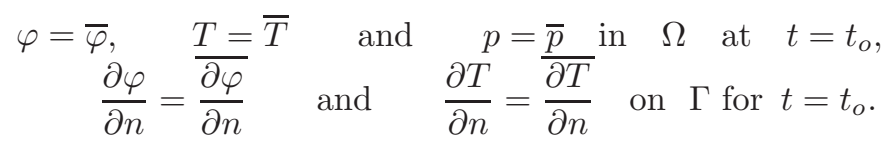

As a rule, the heat and moisture exchange between a solid wall surface and its surrounding can be prescribed by the boundary conditions of the first, second and third kind on the parts of the boundary $\Gamma_{1}, \Gamma_{2}$ and $\Gamma_{3}$, respectively, such that the solution boundary $\Gamma=\Gamma_{1}+\Gamma_{2}+\Gamma_{3}$.

The boundary conditions of the first or Dirichlet kind, where surface conditions are the same as the ambient conditions, are given by the known surface value of the driving potentials:

$$
T=\bar{T} \quad \text { and } \quad \varphi=\bar{\varphi} \quad \text { on } \quad \Gamma_{1} \text { for } t>t_{o},
$$

and this condition can be applied when the building component is in contact with water or earth. In the case of liquid transport, this applies when the component surface is completely wetted by rain or ground water.

Boundary conditions of the second or Neumann type require knowledge of heat or mass flow at the surface and are given by the prescribed surface temperature and relative humidity normal derivative values, respectively:

$$
\frac{\partial T}{\partial n}=\frac{\overline{\partial T}}{\partial n} \quad \text { and } \quad \frac{\partial \varphi}{\partial n}=\frac{\overline{\partial \varphi}}{\partial n} \quad \text { on } \quad \Gamma_{2} \quad \text { for } \quad t>t_{o},
$$

Symmetry conditions and adiabatic or moisture-tight conditions are given by the zero flux condition. 
Boundary conditions of the third or Cauchy type are the most frequent kind of heat and moisture transfer between the component surface and the ambient. Special compatibility and restriction conditions must be satisfied at the boundary surfaces of the heat and moisture transfer region. There must be an energy and moisture balance between the heat/moisture flow within the solid to/from the surface, and the heat/moisture that is leaving/entering the surface.

Let us first consider vapour transfer. The normal total vapour flux $n_{v}=\vec{n}_{v} \cdot \vec{n}=$ $\left(\vec{j}_{v}+\vec{j}_{v, \text { conv }}\right) \cdot \vec{n}$ flowing within the solid from the surface must be equal to vapour transfer from the ambient, denoted by the index $a$, to the solid surface given by the constitutive model as follows:

$$
-n_{v}=\delta_{p} \frac{\partial p_{v}}{\partial n}-j_{v, \text { conv }}=\beta_{p}\left(p_{v, a}-p_{v}\right)-j_{v, \text { conv }, a} \quad \text { on } \quad \Gamma_{3} \text { for } t>t_{o},
$$

where the quantities $\beta_{p}$ and $p_{v, a}$ are the vapour transfer coefficient and ambient vapour pressure, respectively. Eq. (8) can be transformed using relative humidity and the temperature as driving potentials as follows:

$$
-n_{v}=\delta_{p} p_{s} \frac{\partial \varphi}{\partial n}+\delta_{p} \frac{d p_{s}}{d T} \frac{\partial T}{\partial n} \varphi-j_{v, c o n v}=\beta_{p}\left(p_{v, a}-\varphi p_{s}\right)-j_{v, c o n v, a},
$$

yielding an expression for the relative humidity normal derivative

$$
\frac{\partial \varphi}{\partial n}=-\left(\frac{\beta_{p}}{\delta_{p}}+\frac{1}{p_{s}} \frac{d p_{s}}{d T} \frac{\partial T}{\partial n}\right) \varphi+\frac{\beta_{p}}{\delta_{p}} \frac{p_{v, a}}{p_{s}}+\frac{1}{\delta_{p} p_{s}}\left(j_{v, \text { conv }}-j_{v, c o n v, a}\right) .
$$

Based on eq. (10) one may conclude, that in all outflow surfaces vapour convective fluxes cancelled each other, $j_{v, c o n v}=j_{v, c o n v, a}$.

Heat transfer can be treated in the same way. The normal total heat flux $q=\vec{q} \cdot \vec{n}=\left(\vec{q}_{\text {sens }}+\vec{q}_{\text {lat }}+\vec{q}_{\text {conv }}\right) \cdot \vec{n}$ flowing within the solid from the surface must be equal to the heat inflow from the ambient to the solid surface given by the following constitutive model

$$
-q=\alpha\left(T_{a}-T\right)-h_{l a t, a} n_{v}-q_{c o n v, a}+q_{s o l} \text { on } \Gamma_{3} \text { for } t>t_{o},
$$

where the ambient temperature is denoted by $T_{a}$ and the heat transfer coefficient is given by summing the convective and radiation parts $\alpha=\alpha_{\text {con }}+\alpha_{\text {rad }}$. The term $q_{s o l}$ represents heat flow from short-wave solar radiation. Substituting the equation for the sensible heat flow into eq. (11) gives

$$
\begin{array}{r}
-q=\lambda_{\text {eff }} \frac{\partial T}{\partial n}-h_{\text {lat }} n_{v}-q_{\text {conv }} \\
=\alpha\left(T_{a}-T\right)-h_{\text {lat }, a} n_{v}-q_{c o n v, a}+q_{\text {sol }},
\end{array}
$$

yielding an expression for the temperature normal derivative

$$
\begin{array}{r}
\frac{\partial T}{\partial n}=\frac{\alpha}{\lambda_{e f f}}\left(T_{a}-T\right)+\frac{1}{\lambda_{e f f}}\left[q_{\text {sol }}\right. \\
+\left(h_{\text {lat }}-h_{\text {lat }, a}\right) n_{v} \\
\left.+\left(q_{\text {conv }}-q_{\text {conv }, a}\right)\right] .
\end{array}
$$


Again, one may conclude, that on all outflow boundaries, the latent and convective fluxes cancelled each other.

The Dirichlet and Neumann boundary conditions for the pressure equation may be determined for the solution boundary and the following relations are valid

$$
p=\bar{p} \quad \text { on } \quad \Gamma_{1} \quad \text { and } \quad \frac{\partial p}{\partial n}=\frac{\overline{\partial p}}{\partial n}=-\frac{\rho_{a}}{\delta_{a}} v_{n} \quad \text { on } \quad \Gamma_{2},
$$

where $v_{n}=\vec{v} \cdot \vec{n}$ is the normal velocity component.

\subsection{Interface conditions}

The chosen driving potentials for heat and moisture transport in porous media, the relative humidity $\varphi$, the vapour pressure $p_{v}$ and temperature $T$ are continuous field functions at the contact between the two porous materials, therefore the compatibility conditions give us the equalities

$$
T^{(1)} \equiv T^{(2)} \equiv T^{(I)} \quad \text { and } \quad \varphi^{(1)} \equiv \varphi^{(2)} \equiv \varphi^{(I)} \quad \text { on } \quad \Gamma_{I} .
$$

The energy and moisture equilibrium conditions at the interface $\Gamma_{I}$ between $\Omega_{1}$ and $\Omega_{2}$ are given by continuous energy flow $\vec{q}=\vec{q}_{\text {sens }}+\vec{q}_{l a t}$ and continuous moisture flow $\vec{j}=\vec{j}_{v}+\vec{j}_{l}$ across the interface, respectively. The energy equilibrium conditions across the interface can therefore be stated as

$$
\left(\vec{q}_{\text {sens }}+\vec{q}_{\text {lat }}\right)^{(1)} \cdot \vec{n}^{(1)} \equiv-\left(\vec{q}_{\text {sens }}+\vec{q}_{\text {lat }}\right)^{(2)} \cdot \vec{n}^{(2)} \quad \text { on } \quad \Gamma_{I},
$$

or, in the extended form for the temperature normal derivative $(\partial T / \partial n)^{(2)}$

$$
\left(\frac{\partial T}{\partial n}\right)^{(2)}=-\frac{\lambda_{e f f}^{(1)}}{\lambda_{e f f}^{(2)}}\left(\frac{\partial T}{\partial n}\right)^{(1)}+\frac{1}{\lambda_{e f f}^{(2)}}\left[\left(\vec{q}_{l a t} \cdot \vec{n}\right)^{(1)}+\left(\vec{q}_{l a t} \cdot \vec{n}\right)^{(2)}\right]
$$

and the moisture equilibrium conditions across the interface can be written in likewise

$$
\left(\vec{j}_{v}+\vec{j}_{l}\right)^{(1)} \cdot \vec{n}^{(1)} \equiv-\left(\vec{j}_{v}+\vec{j}_{l}\right)^{(2)} \cdot \vec{n}^{(2)} \quad \text { on } \quad \Gamma_{I}
$$

or, in extended expression for the relative humidity normal derivative

$$
\left(\frac{\partial \varphi}{\partial n}\right)^{(2)}=-\frac{D_{\varphi}^{(1)}}{D_{\varphi}^{(2)}}\left(\frac{\partial \varphi}{\partial n}\right)^{(1)}-\frac{1}{D_{\varphi}^{(2)}}\left[\left(D_{T} \frac{\partial T}{\partial n}\right)^{(1)}+\left(D_{T} \frac{\partial T}{\partial n}\right)^{(2)}\right] .
$$

The air flow interface conditions can be given by the following compatibility and equilibrium conditions

$$
p^{(1)} \equiv p^{(2)} \equiv p^{(I)} \quad \text { and } \quad \delta_{a}^{(1)}\left(\frac{\partial p}{\partial n}\right)^{(1)} \equiv-\delta_{a}^{(2)}\left(\frac{\partial p}{\partial n}\right)^{(2)} \quad \text { on } \quad \Gamma_{I} \text {. }
$$




\section{Boundary element numerical model}

\subsection{Integral representation for energy and moisture transport equations}

The differential energy and moisture conservation models written for the primitive field function temperature and relative humidity, respectively, can be written in the following general form [7-9]

$$
L[u]+\frac{\partial b_{j}}{\partial x_{j}}+b=a_{o} \frac{\partial u^{2}}{\partial x_{j} \partial x_{j}}-\frac{\partial u}{\partial t}+\frac{\partial b_{j}}{\partial x_{j}}+b=0,
$$

where the notation $L[\cdot]$ stands for the parabolic diffusion linear operator, $u\left(r_{j}, t\right)$ is an arbitrary field function, and the terms $b_{j}\left(r_{j}, t\right)$ and $b\left(r_{j}, t\right)$ represent nonhomogeneous or source effects due to the nonlinear transport coefficients, convection and production of the conservative field function, respectively, with the following corresponding integral representation [6] written for a time step $\Delta t=t_{F}-t_{F-1}$

$$
\begin{aligned}
c(\xi) u\left(\xi, t_{F}\right)+a_{o} \int_{\Gamma} \int_{t_{F-1}}^{t_{F}} u q^{\star} d t d \Gamma=\int_{\Gamma} \int_{t_{F-1}}^{t_{F}}\left(a_{o} q_{j}+b_{j}\right) n_{j} u^{\star} d t d \Gamma \\
\quad-\int_{\Omega} \int_{t_{F-1}}^{t_{F}} b_{j} q_{j}^{\star} d t d \Omega+\int_{\Omega} \int_{t_{F-1}}^{t_{F}} b u^{\star} d t d \Omega+\int_{\Omega} u_{i, F-1} u_{F-1}^{\star} d \Omega,
\end{aligned}
$$

where $q=\partial u / \partial n=q_{j} n_{j}$ and $u^{\star}\left(\xi, s ; t_{F}, t\right)$ are the field function normal flux and the parabolic diffusion fundamental solution [8], where $s$ is an arbitrary field point in the solution domain or on the boundary, respectively, and $\xi$ is the source point. The nonhomogeneous terms $b_{j}$ and $b$ are given by the following relations, i.e. for the energy transport

$$
\begin{array}{r}
b_{j}=\frac{1}{c_{o}}\left[\widetilde{\lambda} \frac{\partial T}{\partial x_{j}}-h_{l a t} n_{v j}-\left(\rho_{a} c_{p a}+\frac{c_{p v}}{R_{w}} \frac{p_{s}}{T} \varphi\right) v_{j} T\right], \\
b=-\frac{\widetilde{c}}{c_{o}} \frac{\partial T}{\partial t}+\frac{I}{c_{o}},
\end{array}
$$

with $c_{e f f}=c_{o}+\widetilde{c}, \lambda_{e f f}=\lambda_{o}+\widetilde{\lambda}$ and $a_{o}=\lambda_{o} / c_{o}$, and for the moisture transport

$$
b_{j}=\frac{1}{\theta_{o}}\left(\widetilde{D_{\varphi}} \frac{\partial \varphi}{\partial x_{j}}+D_{T} \frac{\partial T}{\partial x_{j}}-D_{l} \rho_{l} g_{j}-\frac{1}{R_{w}} \frac{p_{s}}{T} v_{j} \varphi\right), \quad b=-\frac{\widetilde{\theta}}{\theta_{o}} \frac{\partial \varphi}{\partial t}
$$

and $\theta=\theta_{o}+\widetilde{\theta}, D_{\varphi}=D_{\varphi, o}+\widetilde{D_{\varphi}}$ and $a_{o}=D_{\varphi, o} / \theta_{o}$ 


\subsection{Integral representation for air dynamics}

Employing the linear elliptic Laplace differential operator, the following relation may be considered for the pressure eq. (4)

$$
L[p]+\frac{\partial b_{j}}{\partial x_{j}}=\frac{\partial^{2} p}{\partial x_{j} \partial x_{j}}+\frac{\partial b_{j}}{\partial x_{j}}=0,
$$

with the corresponding boundary-domain integral representation

$$
c(\xi) p(\xi)+\int_{\Gamma} p q^{\star} d \Gamma=\int_{\Gamma} \frac{\partial p}{\partial n} u^{\star} d \Gamma+\int_{\Gamma} b_{j} n_{j} u^{\star} d \Gamma-\int_{\Omega} b_{j} q_{j}^{\star} d \Omega,
$$

where $u^{\star}(\xi, s)$ is the elliptic Laplace fundamental solution [9], whilst the nonhomogeneous nonlinear term $b_{j}$ is given by the expression

$$
b_{j}=\frac{\widetilde{\delta_{a}}}{\delta_{a o}} \frac{\partial p}{\partial x_{j}}
$$

and $\delta_{a}=\delta_{a o}+\widetilde{\delta_{a}}$. For the constant air permeability $\delta_{a}$, the pseudo force term $b_{j} \equiv 0$, and the pressure field is given by the linear Laplace equation represented by the first two boudary integrals in eq. (26).

\subsection{Discretized equations}

For the numerical solution of eq. (22), the boundary $\Gamma$ is discretized into a series of boundary elements and the domain $\Omega$ is discretized into a series of internal cells. Furthermore, field functions and their derivatives are assumed to vary within each element or cell and each time step according to the space $\{\Phi\}$ or $\{\phi\}$ and time $\{\Psi\}$ interpolation functions such that

$$
\begin{aligned}
u(S, t) & =\{\Phi\}^{T}\{\Psi\}\{u\}_{m}^{n}, \quad q(S, t)=\{\Phi\}^{T}\{\Psi\}\{q\}_{m}^{n}, \\
b_{j}(S, t) & =\{\Phi\}^{T}\{\Psi\}\left\{b_{j}\right\}_{m}^{n}, \quad b_{j}(s, t)=\{\phi\}^{T}\{\Psi\}\left\{b_{j}\right\}_{m}^{n}, \quad \text { etc. }
\end{aligned}
$$

where index $n$ refers to the number of nodes within each element or cell, and the index $m$ refers to the degree of variation of the function $\{\Psi\}$. Assuming linear variation of all functions within the individual time increment $\tau=t_{F}-t_{F-1}$, i.e. $m=1,2$ and

$$
\Psi_{1}=\frac{t_{F}-t}{\tau} \quad \text { and } \quad \Psi_{2}=\frac{t-t_{F-1}}{\tau},
$$

the analytical expressions for the time integrals

$$
U_{m}^{\star}=a_{o} \int_{t_{F-1}}^{t_{F}} \Psi_{m} u^{\star} d t, \quad Q_{m}^{\star}=a_{o} \int_{t_{F-1}}^{t_{F}} \Psi_{m} q^{\star} d t
$$


can be derived [14] and eq. (22) can be rewritten as

$$
\begin{aligned}
& c(\xi) u_{2}(\xi)+\sum_{m=1}^{2} \sum_{e=1}^{E}\left[\int_{\Gamma_{e}}\{\Phi\}^{T} Q_{m}^{\star} d \Gamma\right]\{u\}_{m}^{n}=\sum_{m=1}^{2} \sum_{e=1}^{E}\left[\int_{\Gamma_{e}}\{\Phi\}^{T} U_{m}^{\star} d \Gamma\right]\{q\}_{m}^{n} \\
& +\sum_{m=1}^{2} \sum_{e=1}^{E}\left[\int_{\Gamma_{e}}\{\Phi\}^{T} U_{m}^{\star} n_{j} d \Gamma\right]\left\{\frac{b_{j}}{a_{o}}\right\}_{m}^{n}-\sum_{m=1}^{2} \sum_{c=1}^{C}\left[\int_{\Omega_{c}}\{\phi\}^{T} Q_{j m}^{\star} d \Omega\right]\left\{\frac{b_{j}}{a_{o}}\right\}_{m}^{n} \\
& +\sum_{m=1}^{2} \sum_{c=1}^{C}\left[\int_{\Omega_{c}}\{\phi\}^{T} U_{m}^{\star} d \Omega\right]\left\{\frac{b}{a_{o}}\right\}_{m}^{n}+\sum_{c=1}^{C}\left[\int_{\Omega_{c}}\{\phi\}^{T} u_{F-1}^{\star} d \Omega\right]\{u\}_{F-1}^{n}, \quad(31)
\end{aligned}
$$

where the symbols $E$ and $C$ denote the number of boundary elements and internal cells, respectively.

\section{Numerical algorithm}

When dealing with nonlinear transport problems the subdomain technique must be used to apply different constant diffusivities to each subdomain. The second reason for applying the subdomain or multidomain model is to cut the storage and CPU time requirements of the single domain BEM approach [5, 7, 13]. The heat energy, moisture and pressure governing equations represent a coupled nonlinear systems of equations that can only be solved iteratively.

\section{Numerical example}

The benchmark test example [15] deals with the moisture redistribution inside a nonhomogeneous multilayered wall $L_{x}=0.420 \mathrm{~m}$ thick and $L_{y}=0.001 \mathrm{~m}$ tall with capillary-active interior insulation. The wall consists of three layers: brick $\delta_{b}=0.365 \mathrm{~m}$, mortar $\delta_{m}=0.015 \mathrm{~m}$ and insulating material $\delta_{i}=0.040 \mathrm{~m}$, shown in fig. 1 . The multilayerd structure is airtight. Thermal conductivity of the brick and the insulating material differs by a factor of 11 in dry conditions. Initial temperature and relative humidity conditions are both constant all over the wall. At time zero there is a sudden change in temperature and vapour pressure on either side of the wall. The simulation time is 60 days.

The initial hygrothermal conditions of the structure were temperature $T_{o}=$ $25^{\circ} \mathrm{C}$, relative humidity $\varphi_{o}=0.60$. The following boundary conditions of the third kind could be prescribed on the left boundary at $x=0 m$ and $0 \leq y \leq L_{y}$

$$
\begin{array}{r}
T_{a, e}=0.0^{\circ} \mathrm{C} \text { and } \quad \alpha_{e}=25.00 \frac{\mathrm{W}}{\mathrm{m}^{2} K} \quad \text { for } t>0 \\
\varphi_{a, e}=0.8 \text { and } \quad \beta_{p, e}=1.8382 \cdot 10^{-7} \frac{\mathrm{s}}{\mathrm{m}} \quad \text { for } t>0
\end{array}
$$




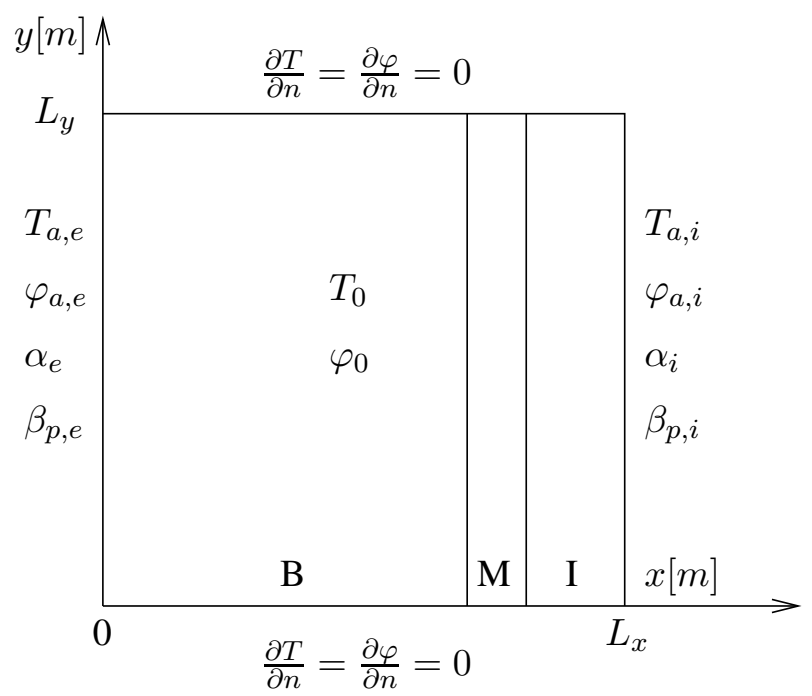

Figure 1: Capillary-active inside insulation: outline of the structure, initial and boundary conditions; $L_{x}=0.420 \mathrm{~m}, L_{y}=0.001 \mathrm{~m}, \delta_{b}=0.365 \mathrm{~m}$, $\delta_{m}=0.015 \mathrm{~m}, \delta_{i}=0.040 \mathrm{~m}$; simulation time 60 days

on the right boundary at $x=L_{x}$ and $0 \leq y \leq L_{y}$

$$
\begin{gathered}
T_{a, i}=20.0^{\circ} \mathrm{C} \quad \text { and } \quad \alpha_{i}=8.00 \frac{\mathrm{W}}{\mathrm{m}^{2} \mathrm{~K}} \quad \text { for } t>0 \\
\varphi_{a, i}=0.6 \text { and } \quad \beta_{p, i}=5.8823 \cdot 10^{-8} \frac{\mathrm{s}}{\mathrm{m}} \quad \text { for } t>0,
\end{gathered}
$$

and zero boundary conditions of the second kind were prescribed on all other boundaries

$$
\frac{\partial \varphi}{\partial n}=0 \quad \text { and } \quad \frac{\partial T}{\partial n}=0 \text { for } t>0 .
$$

The initial conditions were

$$
\varphi_{o}=0.6 \text { and } T_{o}=25^{\circ} \mathrm{C} \text { at } t=0 .
$$

All the transport and materials properties for all three layers are taken from [15] and are given by the following expressions.

The sorption isotherms were given by an expression

$$
W(\varphi)=W_{\text {sat }} \sum_{i=1}^{2} \frac{k_{i}}{\left[1+\left(a_{i} h\right)^{n_{i}}\right]^{m_{i}}}, \quad h=\frac{p_{\text {suc }}}{\rho_{l} g}=-\frac{R_{w} T \ln (\varphi)}{g}
$$


with the exponent $m_{i}=1-1 / n_{i}$. The expressions for vapour permeability $\delta_{p}$ and liquid water permeability $D_{l}$ transport coefficients were given by the expressions

$$
\begin{gathered}
\delta_{p}(W, T)=\frac{D_{v a}}{\mu R_{w} T} \frac{1-\frac{W}{W_{s a t}}}{(1-p)\left(1-\frac{W}{W_{s a t}}\right)^{2}+p}, \\
D_{l}(W)=\exp \left[\sum_{i=0}^{5} a_{i}\left(\frac{W}{\rho_{l}}\right)^{i}\right],
\end{gathered}
$$

and the relations for the effective thermal conductivity and specific heat per volume for dry material, respectively, were given by the relationships

$$
\lambda_{e f f}=\lambda_{m}+\lambda_{m s t} \frac{W}{\rho_{l}} \quad \text { and } \quad c_{p m} \rho_{m}=c_{m} .
$$

Uniform equidistant numerical model of $M 840 \times 1$ macro-elements was considered $(0.5 \mathrm{~mm})$. The time dependent analysis was performed by running the simulation time from the initial state with a time step value of $\Delta=36000,3600$, $360 s$. The conergence criterion was set to $\epsilon=10^{-8}$, and the under-relaxation parameter was set tu $u r=0.5$ for the field functions and the transport properties.

The objective was to calculate the moisture and temperature distributions after $t=60$ days. Grid and time step dependency of the numerical solution was investigated. The moisture distributions between the inside insulation and the mortar layer after 60 days are shown in fig. 2. Solution results for all three time step values match closely with each other and with the other results [15].

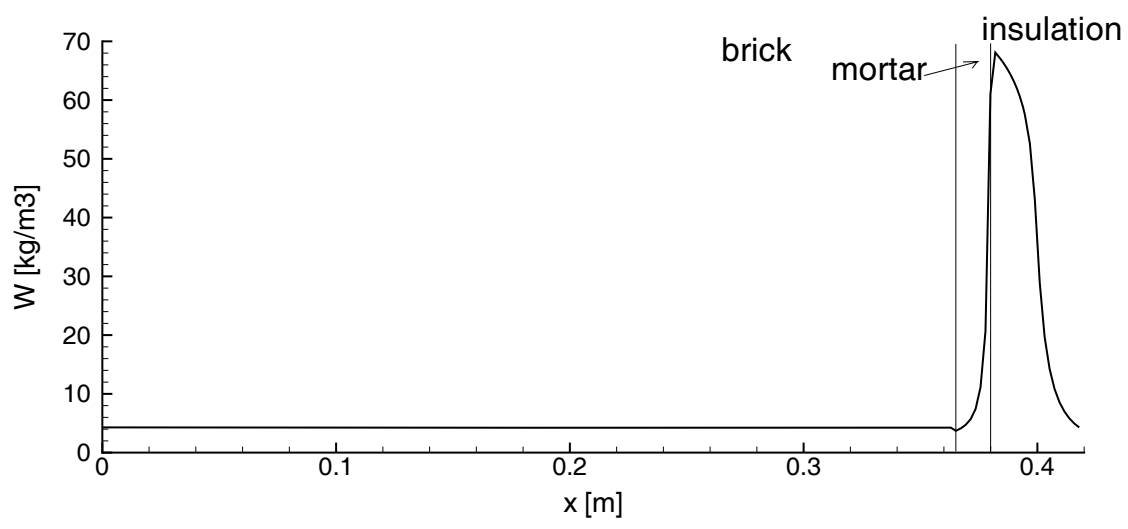

Figure 2: Moisture content profile of the inside insulation and the mortar layer after 60 days. Solution results for all three time step values match closely with each other. 


\section{Conclusions}

The boundary element method has been formulated and implemented to solve the two-dimensional time dependent coupled nonlinear heat, moisture and air flow through a porous solid. Quadratic basis functions were used to approximate the field functions and constant interpolation for fluxes, and the linear variation of all functions over each individual time step was assumed.

One one-dimensional benchmark consisting of moisture redistribution inside a wall with capillary-active interior insulation, was analysed. The good agreement obtained with the respective test case suggests that the simulation model based on a BEM numerical technique can be used to simulate the hygrothermal performance of building envelope components.

\section{Acknowledgements}

The research work presented herein was supported by the Energy and Mobility for Sustainable Regions - EMSURE - Project CENTRO-07-0224-FEDER-002004. This work has also been supported by QREN - Compete under the research project MDFachadas e MDCoberturas - Revestimento em Cortiça para Fachadas e Coberturas Sustentveis e Personalizveis - Project FCOMP-01-0202-FEDER033938.

\section{References}

[1] Hagentoft, C.E.: Intoduction to building physics. Studentlitteratur, Lund (2001).

[2] Tariku, F., Kumaran, K., Fazio, P.: Transient model for coupled heat, air and moisture transfer through multilayered porous media. Int. J. of Heat and Mass Transfer, 53, pp. 3035-3044 (2010).

[3] European standard (EN 15026:2007): Hygrothermal performance of building components and building elements - Assessment of moisture transfer by numerical simulation. pp. 1-24 (2007).

[4] Tadeu, A., Simoes, N., Branco, F.: Steady-state moisture diffusion in curved walls, in absence of condensate flow, via the BEM: a practical Civil Engineering approach (Glaser method). Building and Environment, 38, pp. 677-688 (2003).

[5] Ramšak, M., Škerget, L.: A subdomain boundary element method for highReynolds laminar flow using stream function - vorticity formulation. Int. J. Numer. Meth., 46, pp. 815-847 (2004).

[6] Škerget, L., Brebbia, C.A.: Time dependent non-linear potential problems. Ch. 3: Progress in Boundary Element Methods, Vol.3, Springer-Verlag, Berlin, Heidelberg, New York (1984).

[7] Popov, V., Power, H., Škerget, L.: Domain Decomposition Techniques for Boundary Elements, Application to Fluid Flow. Advances in Boundary Element Series, WIT Press, Southampton, Boston (2007). 
[8] Wrobel, L.C.: The Boundary Element Method, Vol.1, Applications in Thermo-Fluids and Acoustics. John Wiley \& Sons, Ltd, New York (2002).

[9] Brebbia, C.A.: The Boundary Element Method for Engineers. Pentech Press, London (1978).

[10] Škerget, L., Jecl, R.: Boundary element method for transport phenomena in porous medium. Transport Phenomena in Porous Media II, Pergamon (2002).

[11] Hagentoft, C.E., Kalagasidis, A.S. and Adl-Zarrabi, B., Roels,S., Carmeliet, J. and Hens, H., Grunewald, J. and Funk, M., Becker, R. and Shamir, D., Adan. O. and Brocken, H., Kumaran, K. and Djebbar, R.: Assessment method of numerical prediction models for combined heat, air and moisture transfer in building components. Benchmarks for one-dimensional cases. Report.

[12] Ramšak, M., Škerget, L., Hriberšek, M., Žunič, Z.: A multidomain boundary element method for unsteady laminar flow using stream function vorticity equations. Eng. Anal. Bound. Elem., 29, pp. 1-14 (2005).

[13] Ramšak, M., Škerget, L.: A multidomain boundary element method for two equation turbulence models. Eng. Anal. Bound. Elem., 29, pp. 1086-1103 (2005).

[14] Škerget, L., Tadeu, A.: BEM numerical simulation of coupled heat and moisture flow through a porous solid. Eng. Anal. Bound. Elem., 40, pp. 154161 (2014).

[15] Hagentoft, C.E.:HAMSTAD - Final report: Methodology of HAM-Modeling. Report R-02:8. Gothenburg, Department of Building Physics, Chalmers University of Technology (2002). 